\title{
Implementation of an In-situ Infrared Calibration Method for Precise Heat Transfer Measurements on a Linear Cascade
}

\author{
Svenja Aberle ${ }^{1 *}$, Martin Bitter ${ }^{1}$, Florian Hoefler ${ }^{2}$, Jorge Carretero Benignos ${ }^{2}$ and Reinhard Niehuis ${ }^{1}$
}

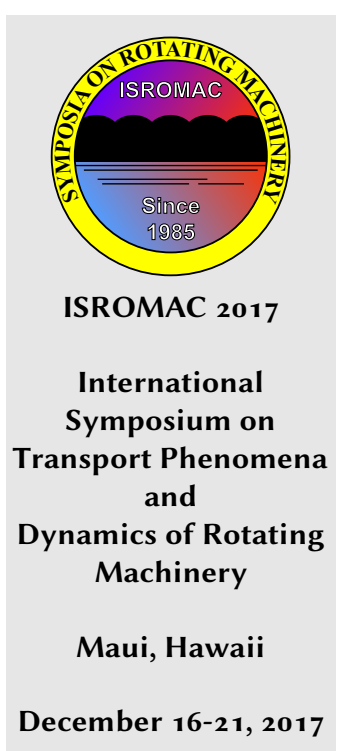

\begin{abstract} surface necessitated the consideration of this dependency. Therefore, a powerful in-situ calibration results, using a local calibration compared to a usual global calibration. By means of these data an evaluation of the aerodynamic behavior of the cascade was enabled. With few modifications, cascade wind-tunnel at the Institute of Jet Propulsion.

\section{Keywords}

Heat Transfer - Linear Cascade - Infrared Imaging - Angular Dependency

${ }^{1}$ Institute of Jet Propulsion, Universität der Bundeswehr München, Neubiberg, Germany

${ }^{2}$ GE Global Research, Garching, Germany

*Corresponding author: svenja.aberle@unibw.de
\end{abstract}

For heat transfer measurements on the center blade of a linear cascade, the infrared measurement technique was set up. As a highly challenging condition the angular dependency of the infrared signal was identified. Beside a shallow angle of view, limited by geometric conditions, the curved blade method was set up, which accounts for the angular dependency implicitly. The correlation of the measured infrared intensity and the temperature was calibrated by a separate calibration function for each position on the blade. In all, three different calibration approaches were proceeded and assessed. Initial measurements in low-speed test conditions delivered physically more reasonable the procedure is capable to deliver high-precision heat transfer measurements in the high-speed

\section{INTRODUCTION}

The aim of emissions reduction and fuel saving is widely spread in all parts of technology, especially in aerospace engineering. In that respect, there are various fields of research concerning the propulsion system of civil and military aircrafts targeting an improvement of the engine's efficiency. One of these fields is the heat management in specific engine components, which has been addressed as an experimental research topic by the Institute of Jet Propulsion (ISA) of the University of the German Federal Armed Forces in Munich for many years. To widen the range of temperature measurement methods in the Institute's test facilities, the infrared measurement technique was established in cooperation with GE Global Research in Garching, Germany.

In the field of flow characterization, the infrared thermography is commonly used for the visualization of boundary layer conditions as described by Grawunder et al. [1]. The estimation of the convective heat transfer is another application that is discussed in detail by Carlomagno et al. [2]. Independent from the way of measurement, a high accuracy of the measured surface temperature is required in order to determine the heat transfer precisely. At the ISA, previous investigations on the heat transfer of blades in a linear cascade were conducted in the high-speed cascade wind tunnel. With this test facility, transonic cascade tests at moderate flow temperatures between 298 and $333 \mathrm{~K}$ can be conducted. So far, thermochromatic liquid crystals were utilized for the determination of the surface temperature distribution as a base of the subsequent calculation of the heat transfer, see Gomes et al. [3]. Another imaging thermography used at the ISA is the application of temperature-sensitive paint, see Bitter [4]. A major disadvantage of both measurement techniques is the limitation to a certain temperature range for commercially available tools. To enable investigations on the heat transfer for higher surface temperatures in the near future, the infrared thermography was implemented based on the later described in-situ calibration method for cascade testing.

This paper focuses on the precise estimation of the heat transfer coefficient on a linear cascade by means of the infrared measurement technique. A measurement procedure is described, which accounts for three major impacts on the infrared data:

1. Ambient conditions, such as the air temperature

2. The influence of an additional window between camera and test object

3. The local emissivity coefficient, including its angular dependency

Whereas there are commonly used correlations available to consider the former aspects, the angular dependency of the emissivity coefficient has been a major challenge in infrared measurements presented in literature, e.g. Sargent et al. [5]. The difficulty arising due to this dependency is 
demonstrated below by example of a linear cascade in lowspeed test conditions. A powerful in-situ calibration method is presented, which takes all mentioned impacts into account, and therefore enables precise heat transfer measurements under optically challenging conditions.

\section{TEST SET-UP}

The object of interest is a linear cascade, tested in the lowspeed wind tunnel at GE Global Research, which is schematically shown in Figure 1. This test facility is used for basic aerodynamic and thermal investigations on specific engine components. With this atmospheric, open wind tunnel a maximum air speed of around $25 \frac{\mathrm{m}}{\mathrm{s}}$ can be realized. The size of the rectangular, open test section is $400 \mathrm{~mm}$ as regards width, and $600 \mathrm{~mm}$ as regards height. In specific, blades with low flow turning are investigated in this case, targeting the determination of the heat transfer coefficient of both, the suction side and the pressure side. For this purpose, a 16 bit FLIR A655sc infrared camera was utilized. The spectral range of this camera is 7.5 up to $14 \mu \mathrm{m}$ providing $30 \mathrm{mK}$ thermal sensitivity.

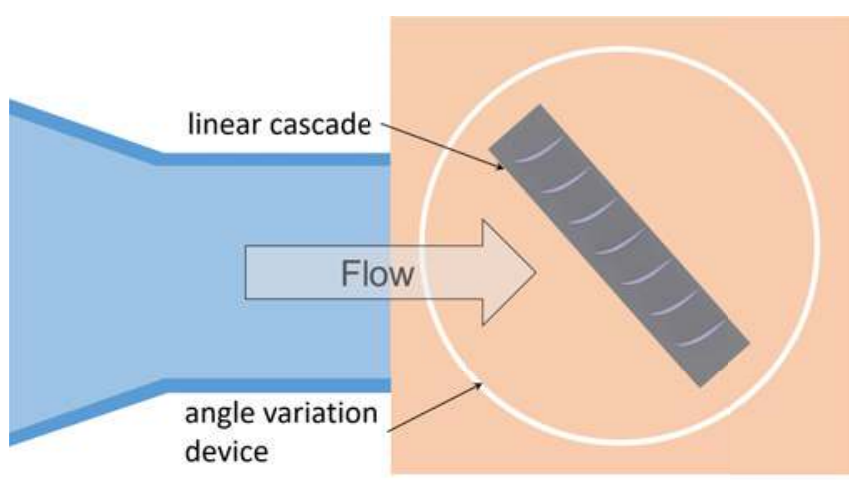

Figure 1. Schematic of cascade wind tunnel; airfoils not to scale

For heat transfer measurements, there are three major tasks to handle:

1. The generation of a heat transfer by a temperature gradient

2. The precise determination of the surface temperature distribution

3. The determination of the heat transfer from the temperature, including heat flux losses

By the first two aspects a suitable modification of the cascade is required. The second point implies the in-situ calibration of the infrared data. Finally, the last point is addressed by an FEM-simulation.

\subsection{Modification of the cascade}

Beside the standard instrumentation such as pressure tab arrays on two of the blades, the cascade was modified specifically for heat transfer measurements. To begin with, the center blade was milled from a low heat-conductive polyurethane to keep conductive losses of the heat flux low. In order to generate the heat transfer an electrical heating of the blade was chosen. For this purpose, an Inconel 600 foil was applied to the surface of interest, either suction side or pressure side, by a thin adhesive film. Both together, the adhesive and the heating foil have a nominal thickness of $75 \mu \mathrm{m}$. At the blade ends the material of the blade was changed to aluminum for two reasons: Firstly, to reach a uniformly distributed electric current, and therefore a constant heat flux over a wide range of the heating foil, and secondly to avoid a heat accumulation inside the sidewalls. The heating foil was finally varnished by a black paint with an emissivity coefficient of about 0.97 . The electrical connection was realized by aluminum clamps that follow the airfoil shape as shown in Figure 2.

For the determination of the temperature distribution on the airfoil, the infrared measurement technique was chosen such that the optical access to the entire blade had to be ensured. Therefore, two germanium windows per side of interest were integrated into one of the sidewalls as also shown in Figure 2. At the respective other side of the blade aluminum plugs were applied for sealing. The tests were conducted with only one infrared camera, switching the camera during the measurement between two defined positions, corresponding to the two windows.

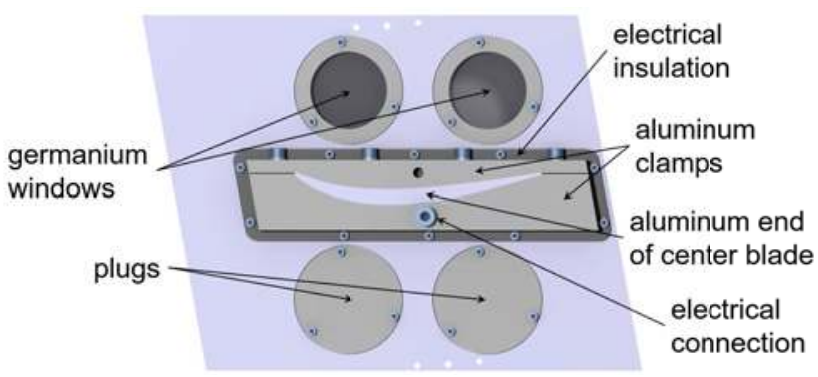

Figure 2. Integration of electrical connectors and optical accesses into the sidewall; airfoil not to scale

\subsection{In-situ infrared calibration}

The temperature data gained by an infrared camera results from a conversion of intensity data. The infrared radiation intensity is measured by a detector as counts, whereas a high spectral radiance is correlated with the intensity.

In understanding typical infrared calibration methods, it is helpful to keep in mind that every atom emits a radiation depending on its temperature. The corresponding wavelength of the thermal radiation is typically within the infrared spectral range. According to Planck's Law, there is a direct correlation between the spectral radiance, the wavelength, and the temperature. Based on this law, an established way of calibration is to correlate the infrared radiation intensity 
$I$ of a black body, with the body temperature $T$ using three calibration constants $R, B$, and $F[6]$ :

$$
I=\frac{R}{e^{B / T}-F}
$$

For a gray body, the emissivity coefficient is usually applied as an additional constant of proportionality. Further correlations are used additionally by camera manufacturers to consider ambient conditions as the air temperature and its emissivity as well as its transmissivity. If applied, the emissivity and transmissivity of a window may have further impacts.

Apart from the calibration by the camera manufacturers there are several approaches known to conduct an insitu calibration. As the accuracy of a calibration is typically higher, the lower the range of its validity is, the calibration directly at the test set-up is potentially capable to deliver highaccuracy infrared temperature measurements, as shown by Schulz [6].

Still, there are two major challenges, which can affect the measurement. Firstly, there are reflections from adjacent parts to be dealt with, which can be avoided in most cases by painting them black or by applying a suitable shielding. Secondly, the angular dependency of the emissivity coefficient has been a limiting constraint in the past for many applications with a shallow angle of view or objects with high curvature. Some approaches, e.g. Sargent et al. [5], present ways how to calibrate the angular dependency, but so far no generally accepted correlation has been found.

In previous tests, the angular dependency was investigated in detail by a particular test set-up using a flat plate. In Figure 3, the measured infrared intensity against the angle of view is shown qualitatively for different temperature levels. The intensity is normalized to the respective intensity at $90^{\circ}$ angle of view. Apart from the fact that the camera detects a lower intensity for lower angles of view, there is an additional correlation of the angular dependency to the temperature level noticeable. Considering this and other effects, an in-situ calibration approach is shown below, which accounts for all mentioned influences on the infrared signal in the same step.

Concerning the presented application, the most challenging condition is the comparably high angular dependency of the emissivity coefficient, especially at shallow angles of view. This one is limited on the one hand by the adjacent blades, and on the other hand by secondary flow close to the sidewalls, which is why a test section in the mid span is preferred. Due to these constraints, the section of measurement was implemented at about $1 / 3$ of the blade span, realizing a maximum angle of view of about $30^{\circ}$. Using an objective lens with a focal length of $13.1 \mathrm{~mm}$, the depth of focus allowed to evaluate an area of $35 \mathrm{~mm}$ of the span. Regarding this area, an especial challenge is the curved blade surface, which implicates that the angle of view towards the blade is variable. Since the mean angle of view is already lower than $30^{\circ}$, the variability of the angle has a noticeable influence, as shown in Figure 3. Thus, a consideration of the

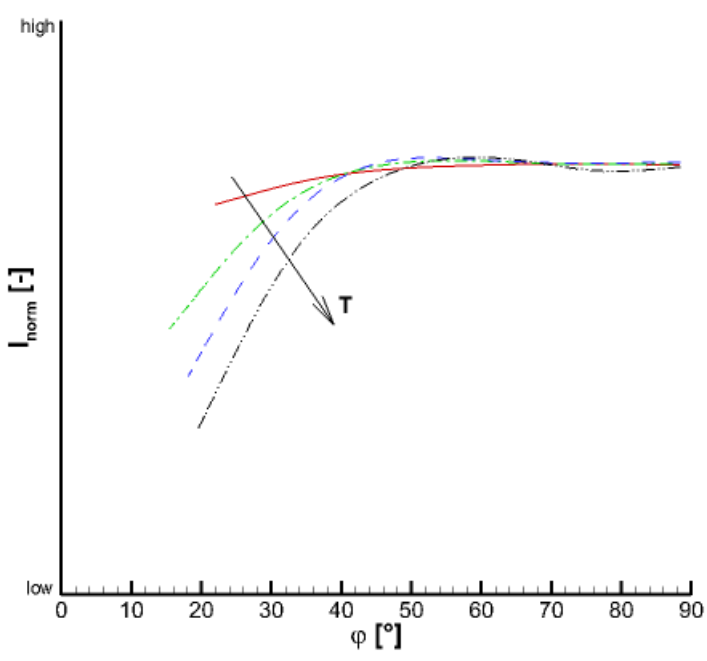

Figure 3. Angular dependency of the infrared signal for four different temperature levels

local emissivity towards the camera position is essential for a high-precision infrared temperature measurement on the cascade. As the separate calibration of the emissivity coefficient considering the angular dependency as well as all ambient conditions is not straight forward, an implicit calibration of the overall intensity-temperature correlation as to Equation 1 was developed.

In particular, the calibration was conducted directly at the test set-up, prior to the measurements. The blade was equipped with PT10o sensors to obtain local reference temperatures. For the initial tests, the data of a maximum number of four temperature sensors could be assimilated. The instrumented blade surface was heated to five temperature levels by means of the heating foil with no flow applied. The corresponding infrared intensity was detected from the same camera positions as during the measurement. This procedure was conducted for the suction side and the pressure side separately. By an established deskewing process, explained in detail by Bitter [4], the intensity data from both camera positions was mapped to a mesh of the corresponding blade surface using about 6500 mesh nodes. Finally, the mean of 45 images for each temperature level was used to apply and compare different calibration processes as described below.

The measured infrared intensity distribution for the highest calibration temperature level is shown in Figure 4a. Apart from a temperature variation along the chord, angular effects are apparent, which is obvious comparing the isolines with the progress of the angle of view towards the blade, shown in Figure 4 b. In comparison, the determined temperature distribution corresponding to the measured intensity is shown in Figure 4c.

In order to allocate the intensity and temperature data, three different approaches were investigated. Apart from a global calibration, which means a single calibration function 


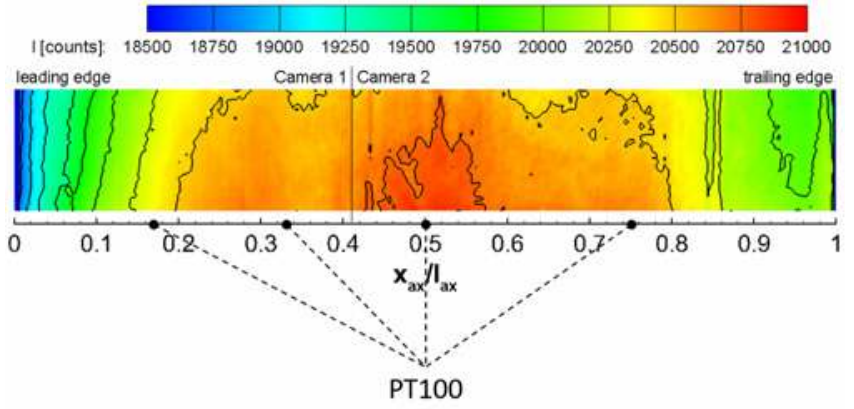

(a) Mapped infrared intensity distribution

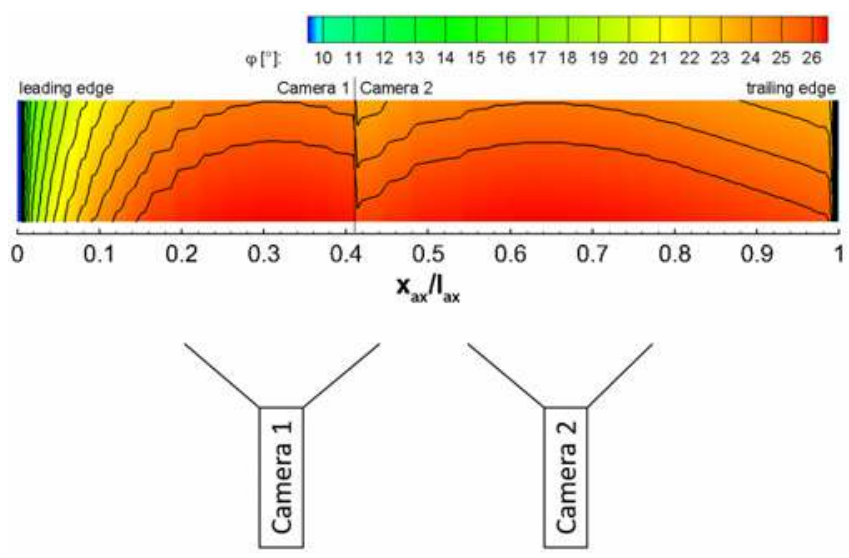

(b) Estimated angle of view

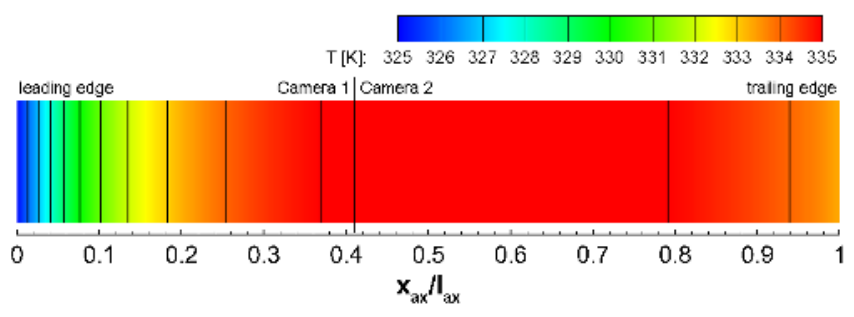

(c) Temperature distribution determined by FEM-simulation

Figure 4. Angular effects of the measured infrared intensity, and allocated temperature distribution for temperature level 5 on the suction side; dimensions not to scale

for the entire blade, two ways of a local calibration were set up, meaning an individual calibration function for each position on the blade, which will be illustrated below.

Global calibration The first and rather common way of calibration is to apply a regression of the measured infrared intensities close to the respective temperature sensor for the different temperature levels. The mean of the four regression functions for the four temperature sensors results in a global calibration function, valid for the entire blade. In Figure 5, the temperatures used for this calibration procedure on the pressure side are schematically marked by a green dashed line. This kind of calibration accounts for ambient conditions, the influence of the germanium windows, and the shallow angle of view. It does not account for the variability of the angle of view.
Local calibration by interpolation As it is obvious that the variable angle of view cannot be considered by only one calibration function for the entire blade surface, a second way of calibration was set up. Whereas the intensity information is available for each position on the blade, respectively each node of the surface mesh used for the mapping, the temperature information is only available at four discrete positions. To receive a temperature distribution from these data, a linear interpolation, or extrapolation respectively, was conducted for all other mesh nodes. The resulting temperature distributions are shown in Figure 5 as a dash-dotted blue line. Afterwards, a local calibration function was set up for each node.

Local calibration by FEM Apparently, the interpolation between only four temperature sensors is a rough approximation. Therefore, a third approach was applied, estimating the temperature distribution by a thermal-electric FEMsimulation by ANSYS 17.2. The local surface temperature is influenced by different types of heat transfer, which is why no constant surface temperature can be realized, despite a constant heating power. First of all, the variable thickness of the blade leads to variable thermal conduction through the blade. Secondly, the inclined blade suffers from variable natural convection, due to buoyancy effects. Lastly, as the first two impacts already cause a variable temperature distribution, the radiative heat flux is an additional variable. However, a twopart FEM-simulation, including the blade and the heating foil was carried out to determine the temperature distribution on the blade. Apart from the electric current applied on the heating foil, there were radiation, conduction, and natural convection considered as boundary conditions on both surfaces. The progress of the heat transfer coefficient for natural convection was estimated by the basic equations for an inclined flat plate as to Vliet [7] and finally calibrated by the measurement data from the four PT1oo sensors. With this approach, a good approximation of the real surface temperature distribution for the different calibration temperature levels was met. It is marked in Figure 5 as a red line. Finally, with this temperature distribution another local calibration for each mesh node was conducted. By this approach, the angular dependency of the emissivity coefficient is considered implicitly additional to other environmental conditions.

\subsection{Determination of the heat transfer coefficient}

The distribution of the heat transfer coefficient $h$ on suction side (SS) and pressure side (PS) is investigated for four different operating points. The definition of the heat transfer coefficient depending on the difference of the wall temperature $T_{w}$ and the adiabatic wall temperature $T_{a}$ is:

$$
h=\frac{q_{c o n v}^{\prime \prime}}{T_{w}-T_{a}}
$$

For low-speed conditions the adiabatic wall temperature is approximately equal to the static inflow temperature. The 


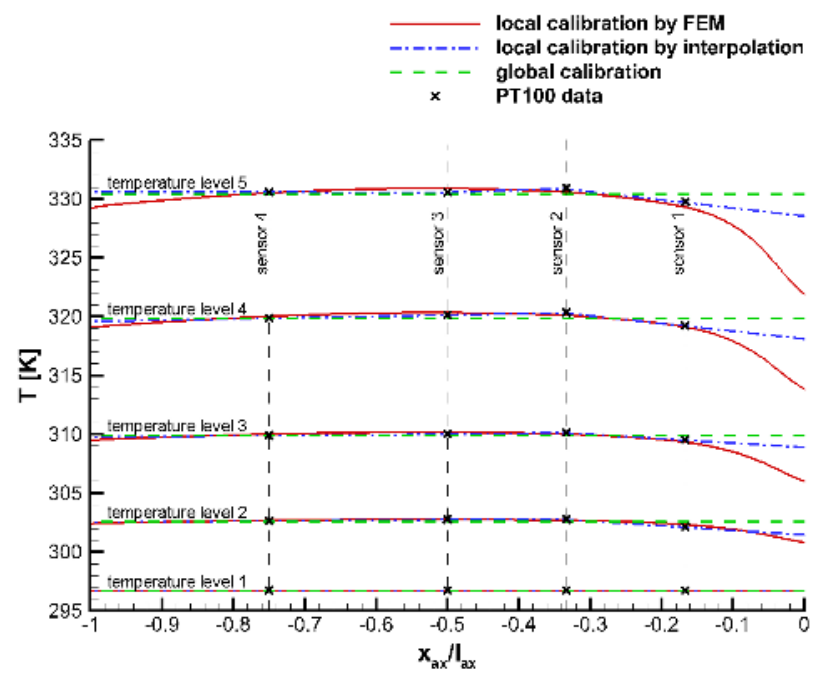

Figure 5. Temperature distributions on the pressure side used for different calibration processes

occurring temperature distribution by heating the blade surface with a constant electric power per conductor cross section $q_{e l}^{\prime \prime}$ is therefore the indicator for the local heat transfer coefficient and the local flow conditions. Apart from the convective heat flux $q_{\text {conv }}^{\prime \prime}$, conductive losses $q_{\text {cond }}^{\prime \prime}$ as well as radiative losses $q_{\text {rad }}^{\prime \prime}$ occur:

$$
q_{e l}^{\prime \prime}=q_{c o n v}^{\prime \prime}+q_{c o n d}^{\prime \prime}+q_{r a d}^{\prime \prime}
$$

Whereas the radiative losses are comparably easy to be determined as far as the temperature distribution is known, the estimation of the conductive losses is a further challenge as these are dependent on the convection occurring at the respective other side of the side of interest. Thus, an iterative approach by use of another FEM-simulation was performed to determine the thermal conduction through the blade, similar as described by Gomes [3].

As illustrated in Figure 6a, the approach starts with the measured temperature distribution on the suction side, which is applied as a load in the thermal simulation. On the pressure side an initial distribution of the heat transfer coefficient is assumed, which results from the infrared measurements without considering any losses. Additionally, thermal radiation was considered on both sides. As a result of this initial FEM-simulation the distribution of the conductive heat flux loss on the suction side can be used to determine a preliminary heat transfer coefficient on the suction side for the following simulation. In this step, the measured temperature distribution on the pressure side is applied as well as the preliminary distribution of the heat transfer coefficient on the suction side, as shown in Figure 6b. The result is the conductive heat flux loss on the pressure side, which can be used for the correction of the pressure side heat transfer coefficient. Continuing this process by several iterations, the distributions of the heat transfer coefficient are obtained.

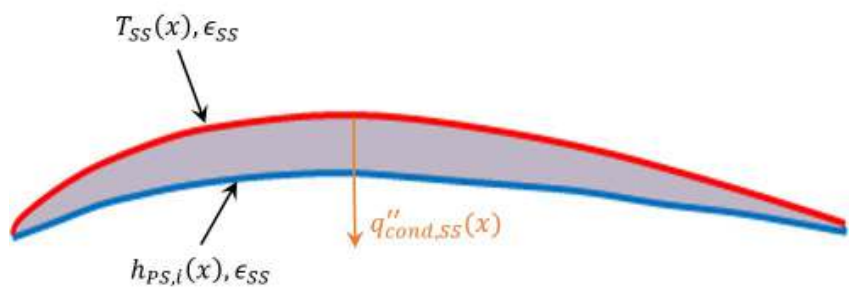

(a) Case: Heated suction side

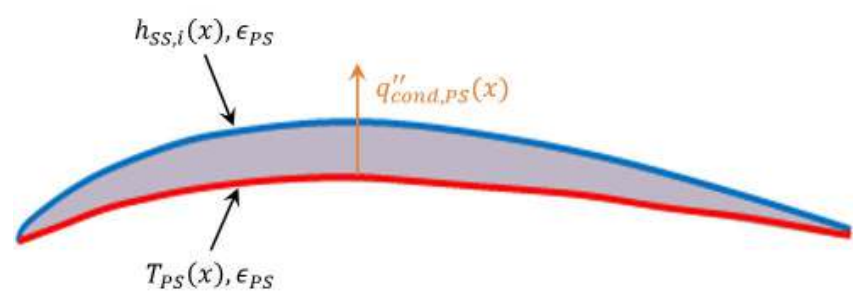

(b) Case: Heated pressure side

Figure 6. Illustration of the FEM-simulation for the calculation of the conductive heat flux loss; airfoil not to scale

\section{RESULTS}

As a result of the described tests, the distribution of the heat transfer coefficient on suction side and pressure side for different flow conditions shown in Table 1 have been investigated. Besides, the in-situ calibration, which was implemented by the ISA for an infrared application for the first time, has to be evaluated.

Table 1. List of investigated operating points

\begin{tabular}{ccc}
\hline operating point & $R e$ & $M a$ \\
\hline 1 & $1.4 \cdot 10^{5}$ & 0.033 \\
2 & $1.7 \cdot 10^{5}$ & 0.038 \\
3 & $1.9 \cdot 10^{5}$ & 0.044 \\
4 & $2.1 \cdot 10^{5}$ & 0.049 \\
\hline
\end{tabular}

\subsection{Evaluation of the in-situ calibration}

Targeting a calibration process, which accounts for angular effects of the infrared signal, two ways of a local calibration were set up and compared to a global calibration function, as outlined above. In order to estimate the accuracy of the methods, the different calibration functions were applied to the intensity data from the calibration images. At the positions of temperature measurement by PT10o sensors, the data was compared to the temperatures determined by infrared data. Applying the local calibration by interpolation, the mean deviation of the temperature was decreased by $0.53 \mathrm{~K}$ compared to the global calibration. The local calibration by FEM allowed for a further decrease of the mean deviation by $0.17 \mathrm{~K}$. In all, for the latter way of calibration, a good correspondence was achieved for sensors 2, 3, and 4, whereas the highest deviation was noted for sensor 1 . This can be ex- 
plained by the fact that the natural convection was estimated by the equations for a flat plate. As the curvature is highest close to the leading edge, where sensor 1 is located, it is reasonable that the calculated temperature distribution shows the highest deviation in this region. For further evaluations of the calibration processes, it has to be considered that their accuracy is comparably high for the calibration points, but there might be higher uncertainties for the distribution of the temperature.

The evaluation of the temperature distributions for the investigated flow conditions emphasizes the importance of a local calibration. In Figure 7, the temperature distribution, normalized to a constant value is exemplarily shown for operating point 1 for both, suction side and pressure side. Regarding the results of the global calibration, it is obvious that angular effects have an influence on the determined results as parts of the progression cannot be explained by effects of the flow. The local calibration approaches, however, lead to a physically more reasonable result. Effects of the boundary layer to the real temperature distribution are clearly separated from angular effects to the measurement, which distort the distribution. Due to the rough approximation of the calibration temperatures for the global calibration as well as the local calibration by interpolation, the local intensity is overinterpreted or underinterpreted respectively, especially close to leading edge and trailing edge. As the local calibration by FEM is based on the most reasonable temperature distribution, it is used for the detailed evaluation of the heat transfer coefficient below.

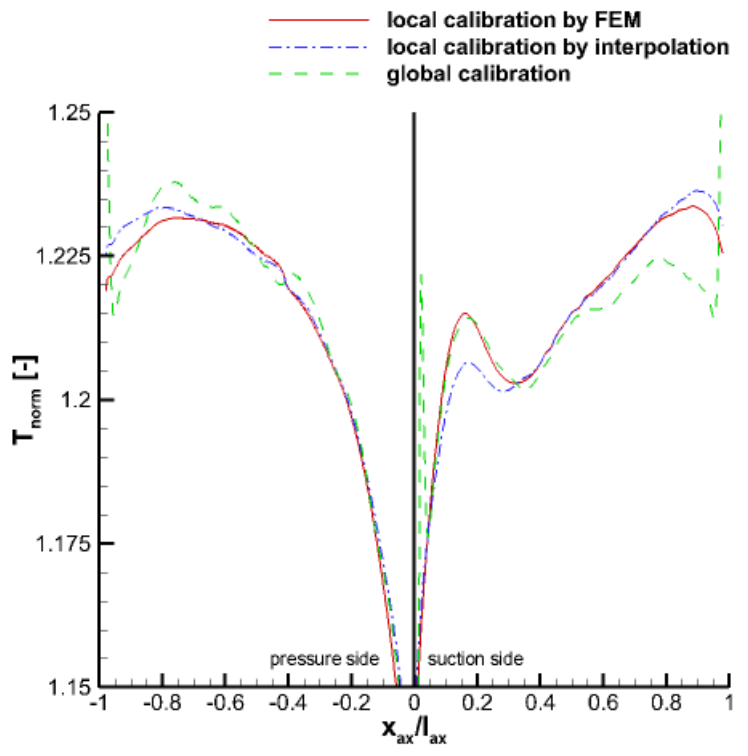

Figure 7. Normalized temperature distribution for operating point 1 , evaluated by different calibration approaches

\subsection{Evaluation of the heat transfer coefficient}

As indicated previously, the consideration of conductive and radiative losses of the heat flux is essential for a precise determination of the heat transfer coefficient. As to the suction side measurement the mean radiative heat flux losses account for about $9 \%$, whereas the amount of the conductive heat flux losses is about $11 \%$. For the pressure side measurement the radiative heat flux losses are about $12 \%$, and the conductive losses about $16 \%$. After considering the heat flux losses, for the four mentioned operating points the heat transfer coefficient was determined as a mean of the measurement area covering $35 \mathrm{~mm}$ of the span. In Figure 8, the distribution of the heat transfer coefficient, normalized to a constant value is shown. Higher Reynolds numbers consequently lead to a higher heat transfer. The distribution of the heat transfer coefficient on suction side and pressure side is typical for airfoils with low flow turning at low-speed conditions. Starting with a laminar boundary layer on the suction side a transition occurs in the leading area of the blade. In contrast to that, no transition is apparent for the pressure side. The transition regions are quantified in Table 2. For higher Reynolds numbers the transition start is located further upstream. Additionally, the relative length of the transition region at operating point 4 indicates that the transition region is smaller for higher Reynolds numbers.

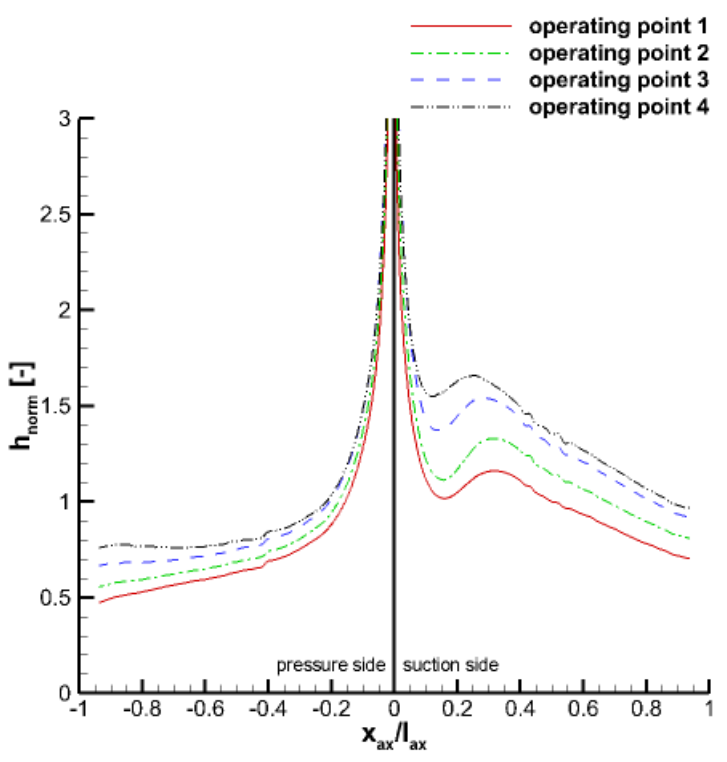

Figure 8. Normalized distribution of the heat transfer coefficient for different flow conditions

Furthermore, the infrared data of 45 images were evaluated statistically. In Figure 9, the standard deviation of the infrared intensity on the suction side is shown. Close to the leading edge, a characteristic peak is apparent for all operating points. Most likely, this can be explained by high fluctuations inside a laminar separation bubble. The enhanced standard deviation is located at the beginning of 
the respective transition region and indicates a transition initiated by a laminar separation bubble.

Table 2. Region of laminar-turbulent transition on the suction side for different operating points

\begin{tabular}{cccc}
\hline $\begin{array}{c}\text { operating } \\
\text { point }\end{array}$ & $\begin{array}{c}x_{a x} / l_{\text {ax }} \\
\text { start }\end{array}$ & $\begin{array}{c}x_{a x} / l_{\text {ax }} \\
\text { end }\end{array}$ & $\begin{array}{c}\text { relative } \\
\text { length }\end{array}$ \\
\hline 1 & 0.16 & 0.33 & 0.17 \\
2 & 0.15 & 0.32 & 0.17 \\
3 & 0.13 & 0.30 & 0.17 \\
4 & 0.11 & 0.26 & 0.15 \\
\hline
\end{tabular}

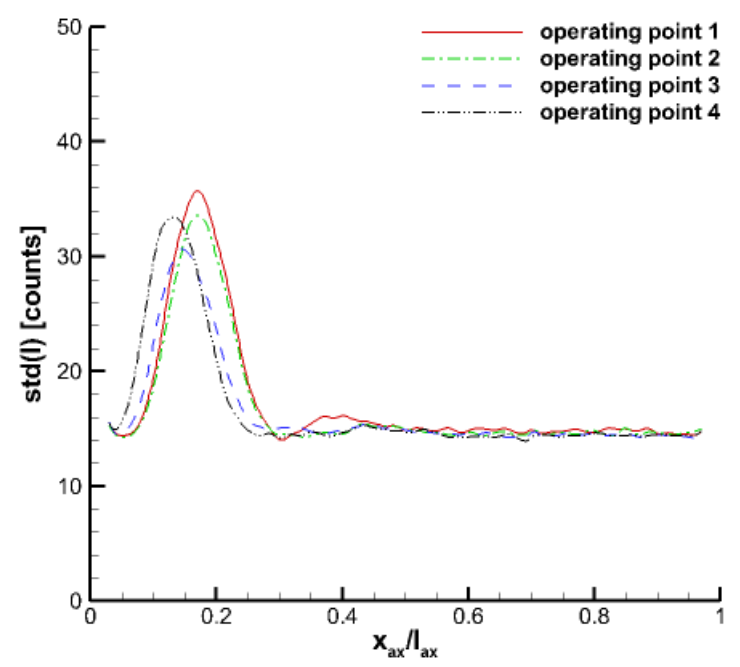

Figure 9. Standard deviation of the measured infrared intensity on the suction side for different operating points

\subsection{Measurement uncertainty}

The accuracy of the results is a consequence of several impacts, which were estimated using error propagation. Primary impacts on the heat transfer coefficient are the accuracy of the heat flux determination and the temperature measurement. The heat flux including all losses is estimated to be determined by $5 \%$ accuracy. The uncertainty of the inflow temperature measurement is $1.5 \mathrm{~K}$, whereas the PT10o sensors have a nominal uncertainty of $0.5 \mathrm{~K}$. Considering further impacts on the calibration as the FEM-simulation, the heat transfer coefficient is estimated to be determined with an accuracy better than about $12 \%$.

\section{CONCLUSIONS AND OUTLOOK}

Targeting high-precision measurements of the heat transfer coefficient by infrared thermography on a linear cascade, a powerful in-situ calibration method was implemented. Apart from specific conditions of the test set-up, this in-situ calibration is capable to account for the angular dependency of the infrared signal. By setting up a local calibration function for each position on the blade, the effect of a variable angle of view towards the curved blade surface is considered implicitly. For this purpose calibration images for several temperature levels were taken to provide a corresponding infrared intensity distribution. In order to obtain suitable temperature distributions for the calibration, different approaches were compared. The most reasonable temperature distributions were achieved by a thermal FEM-simulation. In contrast to a common global calibration, the resulting calibration delivers physically reasonable results. However, for the conducted tests only four temperature sensors were available to control the calibration. To ensure the validity of the calibration for the regions in between the sensor positions, a higher number of local temperature sensors is recommended. Depending on the respective chord length, a determination of the temperature distribution by interpolation instead of the FEM-simulation is expected to be more accurate for a high number of sensors.

Beside the evaluation of the steady state flow condition, a statistical evaluation of the recorded data was conducted, indicating a laminar separation bubble, by peaks of the standard deviation of the infrared intensity, cf. Figure 9. Further information from the comparison of height and width of the peaks is expected from a higher number of images used for the calculation of the standard deviation to achieve a more reliable number of samples.

Finally, the target of the implementation of an infrared measurement system at the ISA is the high-precision measurement of the heat transfer coefficient in the high-speed cascade wind tunnel at high temperatures. As this test facility operates in subatmospheric pressure, a suitable housing for the infrared camera has to be designed. Apart from that, the procedure has to be validated for high-speed test conditions and higher temperature gradients. In all, the presented tests have shown a very promising procedure to obtain high-precision heat transfer data in the high-speed cascade wind-tunnel in the near future.

\section{ACKNOWLEDGMENTS}

The investigations were conducted in cooperation with GE Global Research Europe. The authors would like to thank GE for the permission to publish this data. This project has received funding from the Clean Sky 2 Joint Undertaking under the European Union's Horizon 2020 research and innovation programme under grant agreement No CSJU-CS2GAM-ENG-2014-15. 


\section{NOMENCLATURE}

$B$ calibration coefficient

[K]

$F \quad$ calibration coefficient

$h$ heat transfer coefficient

I infrared intensity

$l \quad$ chord length

Ma Mach number

$q^{\prime \prime}$ heat flux

$R \quad$ calibration coefficient

Re Reynolds number

$T$ temperature

$x \quad$ coordinate parallel to flow direction

$\epsilon \quad$ emissivity coefficient

$\varphi \quad$ angle of view
[6] A. Schulz. Infrared thermography as applied to film cooling of gas turbine components. Measurement Science and Technology, 11(7):948-956, 2000.

[7] G. C. Vliet. Natural convection local heat transfer on constant-heat-flux inclined surfaces. Journal of Heat Transfer, 91(4):511-516, 1969.

\section{Subscripts}

$\begin{array}{ll}a & \text { adiabatic } \\ \text { ax } & \text { axial coordinate } \\ e l & \text { electric } \\ \text { cond } & \text { conductive } \\ \text { conv } & \text { convective } \\ i & \text { iteration number } \\ \text { norm } & \text { normalized } \\ \text { PS } & \text { pressure side } \\ \text { rad } & \text { radiative } \\ S S & \text { suction side } \\ w & \text { wall }\end{array}$

\section{REFERENCES}

[1] M. Grawunder, R. Reß, and C. Breitsamter. Thermographic transition detection for low-speed wind-tunnel experiments. AIAA fournal, 54(6):2012-2016, 2016.

[2] G. M. Carlomagno and G. Cardone. Infrared thermography for convective heat transfer measurements. Experiments in Fluids, 49(6):1187-1218, 2010.

[3] R. Gomes and R. Niehuis. Film cooling effectiveness measurements on highly loaded blades with flow separation. In Proceedings of the 8th European Conference on Turbomachinery, Fluid Dynamics and Thermodynamics, no. 237, 2009.

[4] M. Bitter. Characterization of a turbulent separating, reattaching flow using optical pressure and velocity measurements. Dissertation, Universität der Bundeswehr München, Neubiberg, 2014.

[5] S. R. Sargent, C. R. Hedlund, and P. M. Ligrani. An infrared thermography imaging system for convective heat transfer measurements in complex flows. Measurement Science and Technology, 9(12):1974-1981, 1998. 